\title{
Comparing individual-based and household-based measures of social class to assess class inequalities in women's health: a methodological study of 684 US women
}

Nancy Krieger, Jarvis T Chen, Joseph V Selby

Abstract

Study objective-To describe and compare magnitude of class inequalities in women's health detected with four occupation-based class measures: individual, conventional household (male dominant), gender neutral household, and dominant), gender ne

combined household.
Design-Cross sectional study, using health data obtained by physical examination, laboratory analysis, and self report. Setting-A large pre-paid health maintenance organisation in Oakland, CA (US). Participants-686 women ( $90 \%$ white) enrolled in Examination II of the Kaiser Permanente Women Twins Study (19891990).

Main results-The proportion of women categorised as "working class" equalled 45,30 , and 21 per cent, respectively, for the individual level, gender neutral household, and conventional household class measures. Class inequalities in health, measures. Class inequalities in health,
comparing women categorised as working class with non-working class, generally were stronger using the gender neutral household class measure, compared with the conventional household or individual class measure; in the case of "fair or poor" health, the respective odds ratios and $\mathbf{9 5 \%}$ confidence intervals (adjusted for age and marital status) were $1.9(1.1,3.4)$, $1.5(0.9,2.5)$, and $1.3(0.8,2.2)$, while for high post-load glucose levels, they were $1.7(1.1,2.6), 1.2(0.8,1.7)$, and $1.3(0.9$, $1.8)$. The combined household class measure yielded effect estimates comparable to those of the gender neutral household those of the gender neutral household
class measure but with less precision, class measure but with

Conclusions-Epidemiological studies concerning class inequalities in women's health should use the gender neutral household class measure or, if sample size is sufficiently large, the combined houseis sufficiently large,

(f Epidemiol Community Health 1999;53:612-623) Research Institute, Oakland, USA

JV Selby

Correspondence to: ancy Krieger, Departm f Health and Social Behavior, Harvard School of Huntington Avenue, Boston,
MA 02115, USA.
Analysis of socioeconomic disparities en's health is complicated by debates measure social class of women. Among defining centrality o en's social class remains unresolved. Following what is termed the "conventional" approach, used in the United Kingdom, the United States, and elsewhere, married women typically are assigned the social class of their husband's occupation, based on the presumption that cruption, based on the presumption that econdary wage earners in their households, while single women are assigned their own ocial class. ${ }^{1}{ }^{10}$ Men, by contrast, are assigned their own social class, whether or not they are married.

A combination of women's increasing participation in the paid labour force and contingent rise in dual wage earner households, however, raises questions as to the suitability of the "conventional" approach to measure the social class of women-or, for that matter, men. Fo example, between 1960 and 1990 labour force participation of women in the United States grew from 37.7 to 57.5 per cent, with most of his change attributable to married women's (1) (31.9 per ent in 1960; 58.4 per cent in 1996) (page 403). ${ }^{13}$ Thus, between 1960 and 1990 the proportion of US married couple families consisting of a husband in the labour force and a wife not in the labour force declined from 60.7 to 25.0 per cent, while the proportion comprised of both a husband and wife in the labour force rose from 28.4 to 53.5 per cent (page 820).14 Within 28.4 to 53.5 per cent (page 8 Wroportion of single female headed families increased from 10 to 17 per cent (page 820). ${ }^{14}$

Spurred by women's rising labour force participation, a growing number of social science and public health researchers have begun to challenge the validity of the "conventional" (he wo social class on both theoretical and empirical grounds ${ }^{1-12}$ Historians likewise have cast a critical eye on traditional accounts of class formation centred chiefly on men's work. ${ }^{1516}$ At issue are: (1) the relative importance of individual versus household measures of class, or what US sociologist Erik Olin Wright has termed "direct" and "medited" "class locations (page 223-80), ${ }^{11}$ and (2) different ways of conceptualising and measuring household class. Alternative household class measures proposed include: (a) dominan (based on the most dominant class position represented in the household, regardless of gender), and (b) combined (based on class positions of all working age adults within the ingful measures of women's class position 
sociological research accordingly has examined associations of individual and diverse household class measures with such outcomes as women's subjective class identification, ${ }^{11}$ (page $249-80)^{17-21}$ earnings, ${ }^{2}{ }^{1722}$ voting patterns, ${ }^{18}$ hours spent performing household labour, ${ }^{1}$ (page 281-317) ${ }^{23}$ household food purchasing patterns, ${ }^{17}$ and childbearing patterns. ${ }^{44}$ Most of these studies have reported stronger associations between these diverse outcomes and women's household class (however measured) compared with individual class; a few of the most recent investigations, however, find evidence of increasing effects for married wom-
en's individual level class position. ${ }^{1021}$

en's individual level class position. ${ }^{1021}$ perspective for assessing utility and interpretation of measures of women's social class. Of concern is how different class measures capture pathways by which class inequalities become embodied and manifested as social inequalities in health. ${ }^{25}$ Sensitivity of diverse class measures to socioeconomic disparities in health, for example, may vary depending upon the extent to which risk of a given health outcome is shaped by workplace conditions, household standard of living, and/or economic and social

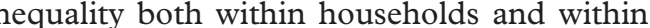
society at large ${ }^{1} 1226-28$

Among the small body of epidemiological research comparing estimates of class inequalities in women's health detected with individual versus household measures of social class, most studies have been conducted in the $\mathrm{UK}^{9}{ }^{29}$ 30-34; $^{\text {; }}$ a handful have been carried out in other countries. ${ }^{35-37}$ These investigations chiefly have compared effect estimates for individual and conventional household measures of social class in relation to mortality and self reported health; like the sociological research, most of these studies have reported stronger class effects for the conventional household class measure. Only two of the epidemiological hold class measure, only two used a combined household class measure, ${ }^{30}$ and none analysed health data obtained by physical examination or laboratory analysis. The purpose of our study therefore was to describe and compare the magnitude of class inequalities in women's health detected with four different occupation based class measures: (a) individual, (b) conventional household (male dominant), (c) dominant (gender neutral) household, and (d) combined household social class, using anthropometric, metabolic, and self report health data from a cohort of adult US women twins.

\section{Methods}

STUDY POPULATION

Women in our cross sectional study were members of a cohort of 352 twin pairs (704 women) who participated in the Kaiser Permanente Women Twins Study Examination II, conducted in 1989-1990 in Oakland, California. ${ }^{38}{ }^{39}$ The original cohort, enrolled at Examination I (1978-1979) consisted of 434 twin pairs (868 women) recruited for a study of coronary heart disease risk factors from a twin registry ( $\mathrm{n}=9821$ pairs $)$ established in 1974 by the Division of Research of the Northern California Region of the Kaiser Permanente Medical Care Program. ${ }^{38} 40$ These 434 twin pairs were recruited from the 642 pairs of women twins identified, through the registry, as residing in the San Francisco Bay Area. Original cohort members thus resided in San Francisco Bay Area at the time of the examination, were born in or before 1960 (mean age $=41$ years; ange: $18-85$ years), and were representative of the women twins in the entire respect to age and race/ethnicity: 90 per cent were white, 7 per cent black, 2 per cent Asian and 1 per cent American Indian. ${ }^{38}$

Follow up for Examination II started 10 years later. Participants in Examination I were ent a self administered questionnaire on their ealth and sociodemogr and were invited to return for physical examination. Cohort retention was high: only 72 women (8.3 per cent) did not respond, of whom 36 were deceased. Among the 796 respondents, only 87 (10.9 per cent) did not return for a physical examination. After excluding five women whose twin was a non-respondent, the final cohort included 704 women (352 twin pairs), representing 81.1 per cent of the original cohort. As in Examination I, 90 per cent of the women in Examination II were white and 7 per cent were black; 3 per cent were Asian. Overall, their mean (SD) age, as expected, was 51 (13.1) 30-44 years old, 43 per cent were 45-64 years old, and 21 per cent were 65 to 91 years old. ${ }^{39}$

Enrollment and study of women in Examination I and II of the Kaiser Permanente Women Twins Study was approved by the Kaier Permanente Medical Care Program,

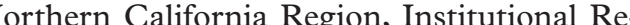
view Board, and conforms to principles embodied in the Declaration of Helsinki. Research conducted for this specific investigation was additionally approved by the Harvard Schoo of Public Health Human Subjects Committee.

\section{DATA COLLECTION}

Socioeconomic data

Respondents provided data on adult socioeconomic characteristics via the self administered mailed questionnaire. Our measures of socia class were based on an empirical class typology developed by US sociologist Erik Olin Wright ${ }^{111}$ and adapted for use in public health research. ${ }^{136}$ Conceptualising social class as a social relation, this typology distinguishes between people who (in order of dominance) are solely in charge of their own labour (that is, own a business and employ others; supervisory employees; self employed), hereafter referred to as "non-working class", (b) work for other as non-supervisory employees - that is, are working class, or (c) are not in the paid labour force. The full schema for Wright's elaborated is presented in ppendix 1 
Individual level social class was based on self reported usual occupational class position. Household level social class was operationalised in three ways: (a) conventional (male dominant/partner defined), equal to the individual level social class of the spouse/partner (hereafter referred to as the "partner"), (b) gender neutral, equal to the most dominant individual level class position (as defined in the preceding paragraph), taking into account the individual level class position of the respondent and her partner or other head of household, if any, and (c) combined, stratifying jointly by the individual level class position of the respondent and her partner or other head of household, if any. ${ }^{1471036}$ Other sociodemographic data obtained from the self administered questionnaire used in this study included: age at Examination II, race/ethnicity, and marital/partner status (with the category "married" including "or living as married"). No data were available on gender of the partner or on the respondent's sexual orientation or identity, precluding distinguishing between heterosexual and lesbian households.

Health outcomes

Health outcomes selected for analysis were chosen because they exhibit well known associations with socioeconomic position, and pertained to diverse outcomes, including overall health status, body build, reproductive history, smoking and physical activity, and cardiovascular profile. ${ }^{27} 4243$ Outcomes based on data from the self administered questionnaire included: age at first pregnancy, self rated health status (excellent, good, fair, or poor), smoking history, and medication use. Using a modified version of the validated one year recall instrument on physical activity used in the Coronary Artery Risk Development in Young Adults (CARDIA) study, physical activity ( $\mathrm{kcal} / \mathrm{kg} / \mathrm{year}$ ) was measured via an interviewer administered questionnaire that assessed typical amount of time spent in activities of varying intensity at home, at work, and during recreation ${ }^{44}{ }^{45}$; values were categorised into quintiles.

Additional anthropometric and metabolic data were obtained by physical examination and laboratory analysis. ${ }^{446}$ Two staff people obtained and recorded the anthropometric measurements, for which participants wore light clothing, shoes removed. Height was recorded to the nearest $0.5 \mathrm{~cm}$, weight was measured to the nearest $0.1 \mathrm{~kg}$, and these data were used to calculate body mass index (BMI, in $\left.\mathrm{kg} / \mathrm{m}^{2}\right)$. Following new WHO guidelines (page 329-30), ${ }^{47} \mathrm{BMI}$ was categorised as $<25$, 25-29.9 ("overweight)", and $\geqslant 30$ ("obese") $\mathrm{kg} / \mathrm{m}^{2}$. Minimal waist girth was measured using a steel measuring tape, either at the natural indentation or, if no indentation was present, at a level midway between the iliac crests and the lower edge of the rib cage. Hip girth was measured at the greatest protrusion of the buttocks. Girths were recorded to the nearest 0.5 $\mathrm{cm}$ and measured twice. Waist to hip ratio was calculated based on average waist and hip girth. Systolic and diastolic blood pressure (seated, right arm) were measured using a mercury sphygomanometer. After a five minute rest period, two measures each of systolic and diastolic blood pressure were taken; av hese two measures were used for data analysis. Women were classified as ever having had high blood pressure if: (a) at Examination II, thei systolic blood pressure was $\geqslant 140 \mathrm{~mm} \mathrm{Hg}$ or their diastolic blood pressure was $\geqslant 90 \mathrm{~mm}$ $\mathrm{Hg}$, and/or (b) they currently were or had ever taken high blood pressure medication.

Blood for lipid and lipoprotein measurement was obtained after participants had fasted overnight and was collected into tubes containng EDTA. Total, high density lipoprotein (HDL), and low density lipoprotein (LDL) cholesterol were measured by standardised methods at the Donner laboratory (Dr R Krauss, University of California, Berkeley, California), a participating laboratory in the Californ Centers for Disease Control lipid standardisation programme. ${ }^{46}$ Total cholesterol concentrations $(\mathrm{mg} / \mathrm{dl})$ were categorised as "desirable" (< 199), "borderline" (200-239), and "high" $\geqslant 240)$; for LDL cholesterol, the range for "desirable," "borderline", and "high" was, respectively, $\leqslant 129,130-159$, and $\geqslant 160$ ours after $75 \mathrm{~g}$ oral glucose load and glucose concentration $(\mathrm{mg} / \mathrm{dl})$ was determined using the glucose oxidase method. ${ }^{44}$ Analyses with glucose concentration excluded 24 women with a self reported history of diabetes; glucose levels were analysed as quintiles.

DATA ANALYSIS

Data analysis entailed four steps. The first consisted of describing the individual social class position of the entire cohort of 704 women and their partners (if any). The second was to quantify proportions of women categorised as "working class" by the individual, conventiona household, and gender neutral household class measures; these analyses were restricted to the 686 women (97.4 per cent of the total cohort) with known data on their individual level social class and that of their partners, if any. Combined household class measures were not included in these analyses, as by definition they do not produce one "summary" measure of household class as "working class" or "nonsole two women who stated they usually were not in the paid labour force and also did not have a partner, the third set of analyses described distributions of health outcomes among the remaining 684 women, stratified by each of the four class measures. Continuous outcomes were analysed as both continuous tions clearly, only categorical analyses are presented (analyses using continuous outcome data available upon request). Descriptive statistics were produced using the statistical package SAS. ${ }^{48}$

Finally, among these same 684 women, we assessed if the magnitude of class inequalities in health varied by class measure by comparing odds ratios from logistic regression models. Each health outcome was dichotomised and 
Table 1 Social class position of each woman and her partner, Kaiser Permanente Women Twins Study (n=704), Oakland, CA, 1989-1990

\begin{tabular}{|c|c|c|c|c|c|c|c|c|c|c|}
\hline \multirow[b]{4}{*}{$\begin{array}{l}\text { Individual's } \\
\text { occupational class }\end{array}$} & \multirow[b]{4}{*}{ Number } & \multirow[b]{4}{*}{ Class location } & \multicolumn{7}{|c|}{ Partner's occupational class } & \multirow[b]{4}{*}{$\begin{array}{l}\text { Total (per cen } \\
\text { of column } \\
\text { total) }\end{array}$} \\
\hline & & & \multicolumn{4}{|c|}{ Class location } & \multirow[b]{3}{*}{ No partner } & \multirow[b]{3}{*}{$\begin{array}{l}\text { Don't } \\
\text { know }\end{array}$} & \multirow[b]{3}{*}{ Missing } & \\
\hline & & & \multicolumn{3}{|c|}{ Non-working class } & \multirow{2}{*}{$\begin{array}{l}\text { Working class } \\
\begin{array}{l}\text { Non-supervisory } \\
\text { employee }\end{array}\end{array}$} & & & & \\
\hline & & & Owner & $\begin{array}{l}\text { Self } \\
\text { employed }\end{array}$ & $\begin{array}{l}\text { Supprvisory } \\
\text { employee }\end{array}$ & & & & & \\
\hline Owner & $\mathrm{n}($ row \%) & $\begin{array}{l}\text { Non-working } \\
\text { class }\end{array}$ & $9(60.0)$ & $2(13.3)$ & $2(13.3)$ & $2(13.3)$ & $0(0)$ & $0(0)$ & $0(0)$ & $15(2.1)$ \\
\hline Self employed & $\mathrm{n}($ row \%) & $\begin{array}{l}\text { Non-working } \\
\text { class }\end{array}$ & $5(11.4)$ & $7(15.9)$ & $15(34.1)$ & $8(18.2)$ & $8(18.2)$ & $0(0)$ & $1(2.3)$ & $44(6.3)$ \\
\hline Supervisory employee & $\mathrm{n}($ row \%) & $\begin{array}{l}\text { Non-working } \\
\text { class }\end{array}$ & $15(4.9)$ & $27(8.9)$ & $132(43.3)$ & $52(17.1)$ & $73(23.9)$ & $6(2.0)$ & $0(0)$ & $305(43.3)$ \\
\hline $\begin{array}{l}\text { Non-supervisory } \\
\text { employee } \\
\text { Not in paid labour }\end{array}$ & $\mathrm{n}($ row \%) & Working class & $11(3.5)$ & $25(7.9)$ & $132(41.8)$ & $76(24.1)$ & $64(20.3)$ & $4(1.3)$ & $4(1.3)$ & $316(44.9)$ \\
\hline & $\mathrm{n}($ row \%) & & $3(14.3)$ & $4(1$ & $9(42)-320$ & $3(14.3)$ & $2(9.5)$ & $0(0)$ & $0(0)$ & $21(3.0)$ \\
\hline $\begin{array}{l}\text { Don't know } \\
\text { Total (per cent of row }\end{array}$ & $\mathrm{n}($ row \%) & & & $1(33.3)$ & $1(33.3)$ & & $1(33.3)$ & $0(0)$ & $0(0)$ & $3(0.4)$ \\
\hline total) & & & $43(6.1)$ & $66(9.4)$ & $29(41.3)$ & $141(20.0)$ & $148(21.0)$ & $10(1.4)$ & $5(0.7)$ & $704(100.0)$ \\
\hline
\end{tabular}

the logit of the probability of the outcome was modelled as a function of (a) class location alone; (b) class location and age (as a continuous variable); and (c) class location, age, and marital status (except for the combined household measure, as by definition it takes into account marital/partner status). For each of these models, odds ratios for class location were calculated setting as referent group women categorised as "non-working class"; in the case of the combined household measure, the referent group was women classified as "non-working class" with partners also classified as "non-working class". To account for the correlation of errors within twin pairs, which would otherwise lead to underestimation of standard errors and overly narrow confidence intervals, ${ }^{49}$ regression parameters were estimated using a generalised estimating equations (GEE) approach with exchangeable correlation structure..$^{51}$ All models were fit using the most recent version of Vincent Carey's implementation of gee () for S-PLUS ${ }^{52}$ and 95 per cent Wald confidence intervals were calculated cent Wald confidence intervals were calculated
based on robust variance estimates. This version of gee() for S-PLUS allows for the correct estimation of the exchangeable working correlation parameter in the presence of singletons (clusters of size one), allowing us to include all eligible twins, whether or not their co-twin was included in the analysis. Analyses restricted to sets of matched twins yielded virtually identical results (data available upon request).

\section{Results}

SOCIAL CLASS DISTRIBUTIONS

Fully 97.4 per cent of the 704 women in the study cohort provided data about their class position, as determined by their usual occupa-

Table 2 Distribution of class locations as classified by individual woman's, gender neutral household, and conventional household class, Kaiser Permanente Women Twins Study

\begin{tabular}{|c|c|c|c|}
\hline & Individual woman & $\begin{array}{l}\text { Gender neutral } \\
\text { household }\end{array}$ & $\begin{array}{l}\text { Conventional } \\
\text { household }\end{array}$ \\
\hline $\begin{array}{l}\text { Working class` } \\
\text { Non-working classt }\end{array}$ & $308(44.9)$ & $143(20.9)$ & $205(29.9)$ \\
\hline Non-working classt $\dagger$ & $357(52.0)$ & $541(78.9)$ & $479(76.2)$ \\
\hline Not in paid labour force & $21(3.1)$ & $2(0.3)$ & $2(0.3)$ \\
\hline
\end{tabular}

${ }^{\star}$ Working class $=$ non-supervisory employee. $\uparrow$ Non-working class $=$ owner, self employed, supervisory employee. tion, and that of their partner (if any). At the time of Examination II, 67 per cent of the women stated they were married or were living as married with their partner, 13 per cent were divorced, 2 per cent were separated, 10 per cent were widowed, and 8 per cent were single.

As shown in table 1, based on the individual level class measure, slightly under half ( 45 per cent) of the 704 women were categorised as working class, slightly over half (52 per cent) as non-working class (43 per cent supervisory employees, 6 per cent self employed, 2 per cent owners of a business and employed others), and only 3 per cent ( 21 women) stated they usually were not in the paid labour force. Fewer than 3 per cent (18 women) had incomplete class data: three women responded "don't know" for their usual occupation, and another 15 did not know $(n=10)$ or report $(n=5)$ their partner's class data.

Among the 686 women who could be assigned a household class measure, the proportion of women categorised as working class by the conventional and gender neutral household class measures was notably lower: 30 and 21 per cent, respectively (table 2 ). Conributing to this differential, both approaches assigned a household class of non-working class to: (a) 55 per cent of the 308 women individually classified as working class, and (b) 76 per cent of the 21 women individually classified as not in the paid labour force. Beyond this, the gender neutral approach assigned a household  class of non-working class to 100 per cent of
the 357 women individually classified as the 357 women individually classified as however, assigned a household class of nonworking class to only 83 per cent of these women.

Lastly, from a combined household class perspective, among these same 686 women, only 11 per cent lived in working class households in which both the respondent and her partner was working class, 9 per cent were single and working class, 31 per cent lived in non-working class households in which both the respondent and her partner was nonworking class, and 12 per cent were single and non-working class (table 1). Additionally, 9 per cent of the women lived in households where 
Table 3 Frequency distributions of health outcomes for all women and by individual and household measures of class, Kaiser Permanente Women Twins Study ( $n=684)$, Oakland, $C A$, 1989-1990

\begin{tabular}{|c|c|c|c|c|c|c|c|c|c|}
\hline \multirow[b]{2}{*}{ Variable } & \multicolumn{2}{|l|}{ All women } & \multicolumn{3}{|c|}{ Working class } & \multicolumn{3}{|c|}{ Non-working class } & \multirow[b]{2}{*}{$\begin{array}{l}\text { Not in paid } \\
\text { labour force } \\
\text { Individual } \\
(n=19) \\
n(\%) \\
n(\%)\end{array}$} \\
\hline & Number* & $\begin{array}{l}(n=684) \\
n(\%)\end{array}$ & $\begin{array}{l}\text { Individual } \\
(n=308) \\
n(\%)\end{array}$ & $\begin{array}{l}\text { Gender } \\
\text { neutral } \\
(n=143) \\
n(\%)\end{array}$ & $\begin{array}{l}\text { Conventional } \\
(n=205) \\
n(\%)\end{array}$ & $\begin{array}{l}\text { Individual } \\
(n=357) \\
n(\%)\end{array}$ & $\begin{array}{l}\text { Gender } \\
\text { neutral } \\
(n=541) \\
n(\%)\end{array}$ & $\begin{array}{l}\text { Conventional } \\
(n=479) \\
n(\%)\end{array}$ & \\
\hline General health rating & 684 & & & & & & & & \\
\hline Excellent & & $252(36.8)$ & $94(30.5)$ & $38(26.6)$ & $64(31.2)$ & $154(43.1)$ & $214(39.6)$ & $188(39.3)$ & $4(21.1)$ \\
\hline Good & & $362(52.9)$ & $177(57.5)$ & $80(55.9)$ & $111(54.2)$ & $173(48.5)$ & $282(52.1)$ & $251(52.4)$ & $12(63.2)$ \\
\hline Fair & & $64(9.4)$ & $35(11.4)$ & $24(16.9)$ & $27(13.2)$ & $26(7.3)$ & $40(7.4)$ & $37(7.7)$ & $3(15.8)$ \\
\hline Poor & & $6(0.9)$ & $2(0.7)$ & $1(0.70)$ & $3(1.5)$ & $4(1.1)$ & $5(0.9)$ & $3(0.6)$ & $0(0)$ \\
\hline Height $(\mathrm{cm})$ & 682 & & & & & & & & \\
\hline$<157.5$ & & $133(19.5)$ & $67(21.8)$ & $32(22.4)$ & $51(25.0)$ & $61(17.2)$ & $101(18.7)$ & $82(17.2)$ & $5(26.3)$ \\
\hline $157.5-161.1$ & & $140(20.5)$ & $65(21.1)$ & $30(21.0)$ & $39(19.1)$ & $73(20.6)$ & $110(20.4)$ & $101(21.1)$ & $2(10.5)$ \\
\hline $161.2-163.9$ & & $119(17.4)$ & $62(20.1)$ & $35(24.5)$ & $41(20.1)$ & $51(14.4)$ & $84(15.6)$ & $78(16.3)$ & $6(31.6)$ \\
\hline $164.0-167.9$ & & $148(21.7)$ & $61(19.8)$ & $30(21.0)$ & $46(22.6)$ & $84(23.7)$ & $118(21.9)$ & $102(21.3)$ & $3(15.8)$ \\
\hline$\geqslant 168.0$ & & $142(20.8)$ & $53(17.2)$ & $16(11.2)$ & $27(13.2)$ & $86(24.2)$ & $126(23.4)$ & $115(24.1)$ & $3(15.8)$ \\
\hline Body mass index $\left(\mathrm{kg} / \mathrm{m}^{2}\right)$ & 682 & & & & & & & & \\
\hline$<25.0$ & & $399(58.5)$ & $182(59.1)$ & $76(53.2)$ & $114(55.9)$ & $207(58.3)$ & $323(60.0)$ & $285(59.6)$ & $10(52.6)$ \\
\hline $25.0-29.9$ & & $157(23.0)$ & $65(21.1)$ & $37(25.9)$ & $53(26.0)$ & $84(23.7)$ & $120(22.3)$ & $104(21.8)$ & $8(42.1)$ \\
\hline$\geqslant 30.0$ & 681 & $126(18.5)$ & $61(19.8)$ & $30(21.0)$ & $37(18.1)$ & $64(18.0)$ & $96(17.8)$ & $89(18.6)$ & $1(5.3)$ \\
\hline Waist to hip ratio & 681 & & & & & & & & \\
\hline $\begin{array}{l}0.63-0.75 \\
0.76-0.84\end{array}$ & & $\begin{array}{l}218(32.0) \\
294(43.2)\end{array}$ & & $\begin{array}{l}37(25.9) \\
57(39.9)\end{array}$ & $\begin{array}{l}64(31.4) \\
74(36.3)\end{array}$ & $116(32.8)$ & $181(33.6)$ & $154(32.3)$ & \\
\hline $\begin{array}{l}0.76-0.84 \\
0.85-1.35\end{array}$ & & $\begin{array}{l}294(43.2) \\
169(24.8)\end{array}$ & $\begin{array}{l}131(42.5) \\
78(25.3)\end{array}$ & $\begin{array}{l}57(39.9) \\
49(34.3)\end{array}$ & $\begin{array}{l}74(36.3) \\
66(32.4)\end{array}$ & $\begin{array}{l}154(43.5) \\
84(23.7)\end{array}$ & $\begin{array}{l}237(44.1) \\
120(23.3)\end{array}$ & $\begin{array}{l}220(46.1) \\
103(21.6)\end{array}$ & $\begin{array}{l}9(47.4) \\
7(36.8)\end{array}$ \\
\hline Age at first pregnancy $(\mathrm{y})$ & 646 & & & & & & & & \\
\hline $\begin{array}{l}\leqslant 17 \\
18-19\end{array}$ & & $\begin{array}{l}23(3.6) \\
46(7.1)\end{array}$ & $\begin{array}{l}11(3.8) \\
26(8.9)\end{array}$ & $\begin{array}{l}7(5.2) \\
15(11.1)\end{array}$ & $\begin{array}{l}10(5.2) \\
18(9.3)\end{array}$ & $\begin{array}{l}10(3.0) \\
19(5.7)\end{array}$ & $\begin{array}{l}16(3.1) \\
31(6.1)\end{array}$ & $\begin{array}{l}13(2.9) \\
28(6.2)\end{array}$ & $\begin{array}{l}2(10.5) \\
1(5.3)\end{array}$ \\
\hline $\begin{array}{l}18-19 \\
20-24\end{array}$ & & $\begin{array}{l}46(7.1) \\
198(30.7)\end{array}$ & $\begin{array}{l}266(8.9) \\
97(33.1)\end{array}$ & $\begin{array}{l}15(11.1) \\
42(31.1)\end{array}$ & $\begin{array}{l}18(9.3) \\
59(30.6)\end{array}$ & $93(27.8)$ & $156(30.5)$ & $139(60.7)$ & $8(42.1)$ \\
\hline $25-29$ & & $184(28.5)$ & $76(25.9)$ & $30(22.2)$ & $53(27.5)$ & $100(29.9)$ & $154(30.1)$ & $131(28.9)$ & $8(42.1)$ \\
\hline$\geqslant 30$ & & $76(11.8)$ & $32(10.9)$ & $11(8.2)$ & $17(8.8)$ & $44(13.2)$ & $65(12.7)$ & $59(13.0)$ & \\
\hline never pregnant & & $119(18.4)$ & $51(17.4)$ & $30(22.2)$ & $36(30.3)$ & $68(20.4)$ & $89(17.4)$ & $83(18.3)$ & $0(0)$ \\
\hline $\begin{array}{l}\text { Do you smoke now? } \\
\text { Current }\end{array}$ & 684 & & & & & & & & \\
\hline $\begin{array}{l}\text { Current } \\
\text { Ex-smoker }\end{array}$ & & $\begin{array}{l}104(15.2) \\
202(29.5)\end{array}$ & $\begin{array}{l}43(14.0) \\
94(30.5)\end{array}$ & $\begin{array}{l}19(13.3) \\
40(28.0)\end{array}$ & $\begin{array}{l}29(14.2) \\
58(28.3)\end{array}$ & $\begin{array}{l}58(16.3) \\
103(28.9)\end{array}$ & $\begin{array}{l}85(15.7) \\
162(29.9)\end{array}$ & $\begin{array}{l}75(15.7) \\
144(30.1)\end{array}$ & $\begin{array}{l}3(15.8) \\
5(26.3)\end{array}$ \\
\hline Never & & $378(55.3)$ & $171(55.5)$ & $84(58.7)$ & $118(57.6)$ & $196(54.9)$ & $294(54.3)$ & $260(54.3)$ & $11(57.9)$ \\
\hline $\begin{array}{r}\text { Physical activity } \\
\text { (kcal/kg/vear) }\end{array}$ & 680 & & & & & & & & \\
\hline $\begin{array}{l}\text { (kcal/kg/yea } \\
740-3793\end{array}$ & & $131(19.3)$ & $65(21.2)$ & $40(28.0)$ & $48(23.4)$ & $52(14.7)$ & $91(17.0)$ & $83(17.5)$ & $14(73.7)$ \\
\hline $3794-5729$ & & $137(20.1)$ & $73(23.8)$ & $30(21.0)$ & $43(21.0)$ & $63(17.8)$ & $107(19.9)$ & $94(19.8)$ & $1(5.3)$ \\
\hline $5730-7149$ & & $137(20.1)$ & $55(17.9)$ & $22(15.4)$ & $35(17.1)$ & $81(22.9)$ & $115(21.4)$ & $102(21.5)$ & $1(5.3)$ \\
\hline $7150-9145$ & & $139(20.4)$ & $64(20.9)$ & $30(21.0)$ & $47(22.9)$ & $74(20.9)$ & $109(20.3)$ & $92(19.4)$ & $1(5.3)$ \\
\hline $9146-25175$ & & $136(20.0)$ & $50(16.3)$ & $21(14.7)$ & $32(15.6)$ & $84(23.7)$ & $115(21.4)$ & $104(21.9)$ & $2(10.5)$ \\
\hline Ever high blood pressure & 684 & & & & & & & & \\
\hline Yes & & $62(9.1)$ & $26(8.4)$ & $18(12.6)$ & $25(12$ & $33(9.2$ & $44(8$. & $37(7.7)$ & $3(15$ \\
\hline $\begin{array}{l}\text { No } \\
\text { Total cholesterol (mg/dl) }\end{array}$ & 683 & $622(90.9)$ & $282(91.6)$ & $125(87.4)$ & $180(87.8)$ & $324(90.8)$ & $497(91.9)$ & $442(92.3)$ & $16(84.2)$ \\
\hline Desirable $(\leqslant 199)$ & & $490(71.7)$ & $212(69.1)$ & $98(69.0)$ & $146(71.6)$ & $267(74.8)$ & $392(72.5)$ & $344(71.8)$ & $11(57.9)$ \\
\hline Borderl & & $141(2$ & $69(22.5)$ & $30(21.1)$ & $39(19.1)$ & $65(18.2)$ & $111(20.5)$ & $102(21.3)$ & $7(36.8)$ \\
\hline High $(\geqslant 240)$ & & $52(7.6)$ & $26(8.5)$ & $14(9$. & $19(9.3)$ & $25(7.0)$ & $38(7.0)$ & $33(6.9)$ & $1(5.3)$ \\
\hline LDL cholesterol (mg/dl) & 683 & & & & & & & & \\
\hline Desirable $(\leqslant 129)$ & & $442(64.7)$ & $187(60.9)$ & $85(59.9)$ & $128(62.8)$ & $247(69.2)$ & $357(66.0)$ & $314(65.6)$ & $8(42.1)$ \\
\hline 159) & & 143 & $73(2$ & $35(2-r) r)(x)$ & $46(2) r) r)(2)$ & $60(16.8)$ & $108(20.0)$ & $97(20.3)$ & $10(52.6)$ \\
\hline High $(\geqslant 160)$ & & $98(14.3)$ & $47(15.3)$ & $22(15.5)$ & $30(14.7)$ & $50(14.0)$ & $76(14.1)$ & $68(14.2)$ & $1(5.3)$ \\
\hline Post-load glucose $(\mathrm{mg} / \mathrm{dl})$ & 638 & & & & & & & $84(18$ & $0(0)$ \\
\hline $\begin{array}{l}<74 \\
74-87.9\end{array}$ & & $\begin{array}{l}120(180.8) \\
129(20.2)\end{array}$ & $\begin{array}{l}47(16.1) \\
54(19.1)\end{array}$ & $\begin{array}{l}25(16.7) \\
17(13.4)\end{array}$ & $\begin{array}{l}36(19.4) \\
33(17.7)\end{array}$ & $\begin{array}{l}73(21.7) \\
73(21.7)\end{array}$ & $\begin{array}{l}95(18.6) \\
112(21.9)\end{array}$ & $\begin{array}{l}84(18.6) \\
96(21.2)\end{array}$ & $\begin{array}{l}0(0) \\
2(11.1)\end{array}$ \\
\hline $88-102.9$ & & $130(20.4)$ & $61(21.6)$ & $26(2$ & $35(18.8)$ & $67(19.9)$ & $104(20.4)$ & $95(21.0)$ & $2(11.1)$ \\
\hline $103-129.9$ & & $129(20.2)$ & $61(21.6)$ & $30(23.6)$ & $44(23.7)$ & $65(19.3)$ & $99(19.4)$ & $85(18.8)$ & $3(16.7)$ \\
\hline$\geqslant 130$ & & $130(20.4)$ & $60(21.2)$ & $29(22.8)$ & $38(20.4)$ & $59(17.1)$ & $101(19.8)$ & $92(20.4)$ & $11(61.1)$ \\
\hline
\end{tabular}

${ }^{\star}$ Total number varied slightly, because of missing data or exclusions. Missing cases did not exceed $6 \%$ of each social class category.

they were non-working class and their partner not in the paid labour force and her partner was was working class; 24 per cent lived in either working class (2.9 per cent) or nonhouseholds where they were working class and working class $(0.1$ per cent).

their partner was non-working class; 2 per cent lived in households where they were not in the

paid labour force and their partner was Table 3 presents data on the overall health pronon-working class, and 0.1 per cent lived in file of the 684 women for whom individual and households where they were not in the paid household level class data were available. labour force and their partner was working Exhibiting better health than US women on class.

Thus, considering only the 539 women with partners, 54 per cent lived in class concordant households ( 14 per cent both working class; 40 per cent both non-working class). Fully 46 per cent, however, lived in class discordant households: 31 per cent where the respondent was working class and her partner was non-working class; 12 per cent where the respondent was non-working class and her partner was working verage (as described more fully in the discussion section), 90 per cent reported their health was excellent or good. Average height equalled $62.5 \mathrm{~cm}$ (SD $6.8 \mathrm{~cm})$, with 21 per cent 168 $\mathrm{cm}$ or taller; average BMI was $25.7 \mathrm{~kg} / \mathrm{m}^{2}$ (SD 5.9), with nearly 40 per cent above the desirable value of $25 \mathrm{~kg} / \mathrm{m}^{2}$; mean waist to hip ratio was $0.80(\mathrm{SD}=0.09)$, with nearly 25 per cent in excess of 0.85 . Among the 82 per cent f wo in

of women reporting having ever been pregnant,
15 per cent were under age 20 and 16 per cent class; and 3 per cent where the respondent was 
Table 4 Frequency distributions of health outcomes by combined household class of women and their partners, Kaiser Permanente Women Twins Study ( $n=665)$, Oakland, $C A, 1989-1990$

\begin{tabular}{|c|c|c|c|c|c|c|}
\hline \multirow[b]{2}{*}{ Individual woman:partne } & \multicolumn{6}{|c|}{ Combined household class } \\
\hline & $\begin{array}{l}\text { NWC:NWC } \\
\left(n^{*}=214\right) \\
n(\%)\end{array}$ & $\begin{array}{l}N W C: W C \\
(n *=62) \\
n(\%)\end{array}$ & $\begin{array}{l}N W C: n o n e \\
\left(n^{\star}=81\right) \\
n(\%)\end{array}$ & $\begin{array}{l}W C: N W C \\
(n *=168) \\
n(\%)\end{array}$ & $\begin{array}{l}W C: W C \\
(n *=76) \\
n(\%)\end{array}$ & $\begin{array}{l}\text { WC:none } \\
\left(n^{*}=64\right) \\
n(\%)\end{array}$ \\
\hline \multicolumn{7}{|l|}{ General health rating } \\
\hline Excellent & $95(44.4)$ & $26(41.9)$ & $33(40.7)$ & $57(33.9)$ & $19(25.0)$ & $18(28.1)$ \\
\hline Good & $100(46.7)$ & $31(50.0)$ & $42(51.9)$ & $97(57.7)$ & $46(60.5)$ & $34(53.1)$ \\
\hline Fair & $17(7.9)$ & $3(4.8)$ & $6(7.4)$ & $13(7.7)$ & $11(14.5)$ & $11(17.2)$ \\
\hline \multirow{2}{*}{\multicolumn{7}{|c|}{ Height $(\mathrm{cm})$}} \\
\hline & & & & & & \\
\hline $\begin{array}{l}<157.5 \\
157.5-161.1\end{array}$ & $46(21.6)$ & $9(14.8)$ & $18(22.2)$ & $35(20.8)$ & $14(18.4)$ & $16(25.0)$ \\
\hline $161.2-163.9$ & $31(14.6)$ & $6(9.8)$ & $14(17.3)$ & $29(17.3)$ & $18(23.7)$ & $15(23.4)$ \\
\hline $164.0-167.9$ & $49(23.0)$ & $16(26.2)$ & $19(23.5)$ & $31(18.5)$ & $13(17.1)$ & $17(26.6)$ \\
\hline \multirow{2}{*}{\multicolumn{7}{|c|}{ Body mass index $\left(\mathrm{kg} / \mathrm{m}^{2}\right)$}} \\
\hline & & & & & & \\
\hline $\begin{array}{l}<25.0 \\
25.0-29.9\end{array}$ & $48(22.5)$ & $16(26.2)$ & $20(24.7)$ & $\begin{array}{l}100(03.1) \\
30(17.9)\end{array}$ & $\begin{array}{l}40(52.6) \\
21(27.6)\end{array}$ & $\begin{array}{l}36(2.3) \\
14(21.9)\end{array}$ \\
\hline$\geqslant 30.0$ & $43(20.2)$ & $7(11.5)$ & $14(17.3)$ & $32(19.1)$ & $15(19.7)$ & $14(21.9)$ \\
\hline \multicolumn{7}{|l|}{ Waist to hip ratio } \\
\hline $0.63-0.75$ & $64(30.2)$ & $27(44.3)$ & $25(30.9)$ & $62(36.9)$ & $18(28.1)$ & $18(28.1)$ \\
\hline $0.76-0.84$ & $100(47.2)$ & $17(27.9)$ & $37(45.7)$ & $75(44.6)$ & $22(34.4)$ & $22(34.4)$ \\
\hline \multirow{2}{*}{\multicolumn{7}{|c|}{ Age at first pregnancy $(y)$}} \\
\hline & & & & & & \\
\hline$\leqslant 17$ & $6(2.9)$ & $3(5.2)$ & $1(1.4)$ & $4(2.5)$ & $6(8.1)$ & $1(1.7)$ \\
\hline $18-19$ & $12(5.9)$ & $3(5.2)$ & $4(5.6)$ & $11(6.8)$ & $9(12.2)$ & $6(10.3)$ \\
\hline $20-24$ & $58(28.4)$ & $17(29.3)$ & $18(25.0)$ & $58(36.0)$ & $20(27.0)$ & $19(32.8)$ \\
\hline $25-29$ & $65(31.9)$ & $23(39.7)$ & $12(16.7)$ & $46(28.6)$ & $22(29.7)$ & $8(13.8)$ \\
\hline $30+$ & $33(16.2)$ & $6(10.3)$ & $5(6.9)$ & $21(13.0)$ & $9(12.2)$ & $2(3.5)$ \\
\hline never pregnant & $30(14.7)$ & $6(10.3)$ & $32(44.4)$ & $21(13.0)$ & $8(10.8)$ & $22(37.9)$ \\
\hline $\begin{array}{l}\text { Do you smoke now? } \\
\text { Current }\end{array}$ & \multicolumn{6}{|c|}{ Do you smoke now? } \\
\hline Ex-smoker & $61(28.5)$ & $18(29.0)$ & $24(29.6)$ & $55(32.7)$ & $18(23.7)$ & $21(32.8)$ \\
\hline Never & $123(57.5)$ & $34(54.8)$ & $39(48.2)$ & $88(52.4)$ & $52(68.4)$ & $31(48.4)$ \\
\hline \multicolumn{7}{|c|}{ Physical activity (kcal/kg/year) } \\
\hline $740-3793$ & $38(18.0)$ & $8(12.9)$ & $6(7.4)$ & $28(16.8)$ & $25(32.9)$ & $12(18.8)$ \\
\hline 3794-5729 & $35(16.6)$ & $13(21.0)$ & 15( & $43(25.8)$ & $17(22.4)$ & $13(20.3)$ \\
\hline 5730-7149 & $46(21.8)$ & $13(21.0)$ & $22(27.2)$ & $33(19.8)$ & $11(14.5)$ & $11(17.2)$ \\
\hline $7150-9145$ & $42(19.9)$ & $17(27.4)$ & $15(18.5)$ & $34(20.4)$ & $12(15.8)$ & $18(28.1)$ \\
\hline \multirow{2}{*}{\multicolumn{7}{|c|}{ Ever high blood pressure }} \\
\hline & & & & & & \\
\hline $\begin{array}{l}\text { Yes } \\
\text { No }\end{array}$ & $\begin{array}{l}17(7.9) \\
197(92.1)\end{array}$ & $\begin{array}{l}7(11.3) \\
55(88.7)\end{array}$ & $\begin{array}{l}9(11.1) \\
72(88.9)\end{array}$ & $159(94.6)$ & $66(86.8)$ & $\begin{array}{l}7(10.9) \\
57(89.1)\end{array}$ \\
\hline \multicolumn{7}{|l|}{ Total cholesterol (mg/dl) } \\
\hline Desirab & $158(73.8)$ & $48(77.4)$ & $61(75.3)$ & $117(69.6)$ & $48(64.0)$ & $47(73.4)$ \\
\hline (200-239) & $41(19.2)$ & $9(14.5)$ & $15(18.5)$ & $39(23.2)$ & $17(22.3)$ & $13(20.3)$ \\
\hline High $(\geqslant 240)$ & $15(7 . c$ & $5(8.1)$ & $5(6.2)$ & $12(7$ & $10(13.3)$ & $4(6.3$ \\
\hline \multicolumn{7}{|l|}{ LDL cholesterol $(\mathrm{mg} / \mathrm{dl})$} \\
\hline Desirable $(\leqslant 129)$ & $148(69.2)$ & $43(69.4)$ & $56(69.1)$ & $104(61.9)$ & $44(58.7)$ & $39(60.9)$ \\
\hline Borderline (130-159) & $35(16.4)$ & $11(17.7)$ & $14(17.3)$ & $39(23.2)$ & $18(24.0)$ & $16(25.0)$ \\
\hline \multirow{2}{*}{\multicolumn{7}{|c|}{$\begin{array}{l}\text { High }(\geqslant 160) \\
\text { Post-load glucose }(\mathrm{mg} / \mathrm{dl})\end{array}$}} \\
\hline & $43(21.4)$ & $11(18.6)$ & $19(24.7)$ & & $10(14.9)$ & $15(25.9)$ \\
\hline $74-87.9$ & $36(17.9)$ & $16(27.1)$ & $21(27.3)$ & $37(23.4)$ & $9(13.4)$ & $8(13.8)$ \\
\hline $88-102.9$ & $46(22.9)$ & $9(15.3)$ & $12(15.6)$ & $35(22.2)$ & $15(22.4)$ & $11(19.0)$ \\
\hline $103-12$ & t & 14( & & & $18(26.9)$ & 12 \\
\hline$\geqslant 130$ & $36(17.9)$ & $9(15.3)$ & $14(18.2)$ & $33(20.9)$ & $15(22.4)$ & $12(20.7)$ \\
\hline
\end{tabular}

${ }^{\star}$ Number varied slightly, because of missing data or exclusions. Missing cases did not exceed $11 \%$ of each social class category. WC $=$ working class, $\mathrm{NWC}=$ non-working class.

were age 30 or older at their first pregnancy. Over half the women ( 55 per cent) had never smoked cigarettes; among the remaining ever smokers, 39 per cent had smoked for 20 years or more and 30 per cent were currently ex smokers. Average annual physical activity was on the order of 6745 (SD 3288) kcal/kg/year. Mean systolic and diastolic blood pressure were, respectively, 118.7 (SD 20.2) and 65.5 (SD 11.3) $\mathrm{mm} \mathrm{Hg}$; only 10 per cent were categorised as ever having high blood pressure. Average total, HDL, and LDL cholesterol equaled, respectively, 181.2 (SD 34.9), 62.9 (SD 16.8) and 120.1 (SD 38.2) mg/dl; for each measure, 60 per cent or more had levels in the "desirable" range. Lastly, among non-diabetic women, mean post-load glucose concentration equalled 106.7 (SD 50.1) mg/dl, of whom 20 per cent had levels in excess of $130 \mathrm{mg} / \mathrm{dl}$.
CLASS INEQUALITIES IN HEALTH: COMPARISONS ACROSS CLASS MEASURES

Distributions of health outcomes

Women classified as "non-working class", regardless of which measure was used, had imilar distributions of health outcomes (table 3). By contrast, women classified as "working class" by the gender neutral household class measure and, to a less degree, by the conventional household measure, were more likely to have a poorer health profile than women classified as "working class" by the individual level measure (table 3). Specifically, they were more likely to: have fair or poor health; be short (in the bottom two height quintiles); have above desirable BMI; have a waist to hip ratio $\geqslant 0.85$; have been pregnant before age 20; be more sedentary (in the bottom two quintiles for physical activity); and 
ever have had high blood pressure. The only outcomes similarly distributed among "working class" women, regardless of class measures, were: current smoking status; undesirable total and LDL cholesterol levels (borderline plus high); and post-load glucose levels. The worst health profile, for each health outcomes, occurred among the 19 women classified as "not in the labour force" (applicable only for the individual level class measure). Data for these 19 women are omitted in subsequent tables, because small numbers precluded meaningful analyses of their health in relation to diverse class measures (data available upon request).

Finer stratification of women using the combined household measure revealed additional patterns, as shown among the 665 women identified as in the paid labour force (table 4). Thus, among women individually classified as "non-working class", those with non-working class partners were least likely to be short and most likely to be in the top height quintile as well as to have had their first pregnancy at age 30 or older; those with working class partners were least likely to be obese; and those with no partner were most likely never to have been pregnant. Among women individually classipregnant. "wmong wo", those with nonworking class partners generally had distribuworking class partners generally had distribu-
tions of health outcomes like those of women tions of health outcomes like those of women individually categorised as non-working class, report excellent health and more likely to be short. Women individually classified as "work-

\begin{abstract}
KEY POINTS
- Studies of class inequalities in women's health are complicated by disagreement over how to measure women's class location: as individuals or in the context of their households.

- We studied associations between women's health and four different class measures: individual, conventional household (male dominant), gender neutral household, and combined household class.

- Class inequalities in health, comparing working class to non-working class women, generally were strongest using women, generally were strongest using
the gender neutral household class measure.

- Epidemiological studies on class inequalities in women's health should use a gender neutral household class measure.

- Future research should examine whether these findings are applicable to men, and also whether results vary by race/ ethnicity, sexual identity, and age (including historical cohort).
\end{abstract}

ng class", however, who had working class partners or no partners had comparably poore health status.

Estimates of class effects

Class inequalities in health comparing women classified as "working class" to "non-working class" were generally strongest for the gender Table 5 Odds ratios $(O R)$ and 95\% confidence intervals (CI)* comparing health outcomes of working class to
non-working class women, using individual and household class measures, Kaiser Permanente Women Twins Study $(n=665)$, Oakland, $C A, 1989-1990$

\begin{tabular}{|c|c|c|c|c|}
\hline & \multirow[b]{2}{*}{$\begin{array}{l}\text { Non-working } \\
\text { class (referent } \\
\text { group) }\end{array}$} & \multicolumn{3}{|l|}{ Odds ratio } \\
\hline & & $\begin{array}{l}\text { Individual working } \\
\text { class } \\
\text { OR }(95 \% \text { CI) }\end{array}$ & $\begin{array}{l}\text { Gender neutral } \\
\text { woorking class } \\
\text { OR ( } 95 \% \text { CI) }\end{array}$ & $\begin{array}{l}\text { Conventional } \\
\text { wooring class } \\
\text { OR }(95 \% \text { CI) }\end{array}$ \\
\hline \multicolumn{5}{|l|}{ General health rating (fair/poor $v$ excellent/go } \\
\hline $\begin{array}{l}\text { crude } \\
\text { adjusted for age and marital/partner status }\end{array}$ & 1.0 & $\begin{array}{l}1.35(0.82,2.23) \\
1.32(0.76 .223)\end{array}$ & $\begin{array}{l}2.07(1.16,3.70) \\
1.94(1.11,3.38)\end{array}$ & $\begin{array}{l}1.67(0.97,2.88) \\
1.51(0.90,2.54)\end{array}$ \\
\hline \multicolumn{5}{|l|}{ Height $(<164 \mathrm{~cm} v \geqslant 164 \mathrm{~cm})$} \\
\hline crude & 1.0 & $1.35(1.03,1.77)$ & $1.37(0.99,1.91)$ & $1.21(0.94,1.56)$ \\
\hline \multirow{2}{*}{\multicolumn{5}{|c|}{ Body mass index $\left(\geqslant 25 \mathrm{~kg} / \mathrm{m}^{2} v<25 \mathrm{~kg} / \mathrm{m}^{2}\right)$}} \\
\hline & & & & \\
\hline $\begin{array}{l}\text { crude } \\
\text { adjusted for age and marital/partner status }\end{array}$ & $\begin{array}{l}1.0 \\
1.0\end{array}$ & $\begin{array}{l}0.98(0.73,1.30) \\
0.96(0.72,1.28)\end{array}$ & $1.25(0.91,1.73)$ & $\begin{array}{l}1.24(0.93,1.65) \\
1.19(0.88,1.61)\end{array}$ \\
\hline \multicolumn{5}{|c|}{ 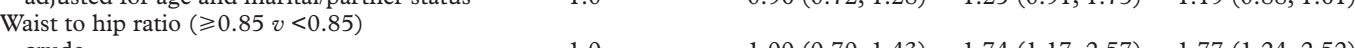 } \\
\hline crude & 1.0 & $1.00(0.70,1.43)$ & $1.74(1.17,2.57)$ & $1.77(1.24,2.52)$ \\
\hline $\begin{array}{l}\text { adjusted for age and marital/partner status } \\
\text { Age at first pregnancy }(<20 \text { vears } \geqslant 20 \text { vears }\end{array}$ & 1.0 & $0.96(0.65,1.42)$ & $1.51(1.00,2.30)$ & $1.60(1.08,2.35)$ \\
\hline \multicolumn{5}{|l|}{ Age at first pregnancy ( $<20$ years $v \geqslant 20$ years) } \\
\hline adjusted for age and marital/partner status & 1.0 & $1.33(0.83,2.14)$ & $1.80(1.04,3.13)$ & $1.65(1.02,2.66)$ \\
\hline \multicolumn{5}{|c|}{ 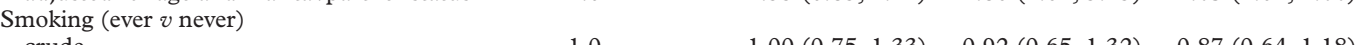 } \\
\hline & 1.0 & $1.00(0.75,1.33)$ & $0.92(0.65,1.32)$ & $0.87(0.64,1.18)$ \\
\hline \multirow{2}{*}{\multicolumn{4}{|c|}{ Physical activity $(<5730 \mathrm{kcal} / \mathrm{kg} / \mathrm{y} v \geqslant 5730 \mathrm{kcal} / \mathrm{kg} / \mathrm{y})$}} & $0.82(0.60,1.12)$ \\
\hline crude & 1.0 & & & \\
\hline adjusted for age and marital/partner status & 1.0 & $1.66(1.16,2.38)$ & $1.69(1.07,2.69)$ & $1.22(0.84,1.78)$ \\
\hline \multicolumn{5}{|l|}{ Ever high blood pressure (yes $v$ no) } \\
\hline & 1.0 & $0.90(0.52,1.58)$ & $1.42(0.76,2.68)$ & $1.42(0.81,2.50)$ \\
\hline \multicolumn{4}{|l|}{ Total cholesterol $(<200 \mathrm{mg} / \mathrm{dl} v \geqslant 200 \mathrm{mg} / \mathrm{dl})$} & 1.20 \\
\hline crude & 1.0 & $1.32(0.95,1.85)$ & $1.18(0.82,1.68)$ & $1.03(0.73,1.46)$ \\
\hline \multirow{2}{*}{\multicolumn{5}{|c|}{$\mathrm{LDL}$ cholesterol $(<130 \mathrm{mg} / \mathrm{dl} v \geqslant 130 \mathrm{mg} / \mathrm{dl})$}} \\
\hline & 1.0 & $1.43(1.05,1.97)$ & $1.29(0.91,1.82)$ & $1.19(0.87,1.65)$ \\
\hline \multirow{2}{*}{\multicolumn{5}{|c|}{ Post-load glucose $(<88 \mathrm{mg} / \mathrm{dl} v \geqslant 88 \mathrm{mg} / \mathrm{dl})$}} \\
\hline & 1.0 & $1.33(0.97,1.83)$ & $1.37(0.93,2.03)$ & $1.15(0.79,1.57)$ \\
\hline adjusted for age and marital/partner status & 1.0 & $1.29(0.92,1.82)$ & $1.67(1.07,2.60)$ & $1.18(0.81,1.73)$ \\
\hline
\end{tabular}



Table 6 Odds ratios $($ OR) and $95 \%$ confidence intervals $(C I) *$ for the associations between selected health outcomes and combined household class, $f$
Kaiser Permanente Women Twins Study $(n=665)$, Oakland, $C A, 1989-1990$

\begin{tabular}{|c|c|c|c|c|c|c|}
\hline \multirow[b]{2}{*}{ Individual woman:partner } & \multicolumn{6}{|c|}{ Combined household class } \\
\hline & $\begin{array}{l}N W C: N W C \\
(n=214) \\
O R(95 \% C I)\end{array}$ & $\begin{array}{l}N W C: W C \\
(n=62) \\
O R(95 \% C I)\end{array}$ & $\begin{array}{l}N W C: \text { none } \\
(n=81) \\
O R(95 \% C I)\end{array}$ & $\begin{array}{l}W C: N W C \\
(n=168) \\
O R(95 \% C I)\end{array}$ & $\begin{array}{l}W C: W C \\
(n=76) \\
O R(95 \% C I)\end{array}$ & $\begin{array}{l}\text { WC:none } \\
(n=64) \\
\text { OR }(95 \% C I)\end{array}$ \\
\hline \multicolumn{7}{|l|}{ General health rating (fair/po } \\
\hline & 1.00 & $0.86(0.30,2.48)$ & 1.94) & $0.93(0.46,1.90)$ & $1.56(0.71,3.43)$ & $1.97(0.78,4.95)$ \\
\hline usted & 1.00 (reference) & $0.72(0.25,2.12)$ & $0.79(0.33,1.92)$ & $0.88(0.42,1.86)$ & $1.25(0.58,2.68)$ & $2.10(0.82,5.35)$ \\
\hline \multicolumn{7}{|l|}{ Height $(<164 \mathrm{~cm} v \geqslant 164 \mathrm{~cm})$} \\
\hline crude & 1.00 (reference) & $1.04(0.70,1.55)$ & $0.99(0$ & $1.28(0$ & $2,2.67)$ & $1.32(0.78,2.23)$ \\
\hline \multicolumn{6}{|c|}{ Body mass index $\left(\geqslant 25 \mathrm{~kg} / \mathrm{m}^{2} v<25 \mathrm{~kg} / \mathrm{m}^{2}\right)$} & $1.32(0.76,2.30)$ \\
\hline crude & 1.00 (reference) & $1.04(0.64,1.70)$ & $1.18(0.76,1.82)$ & $0.91(0.63,1.32)$ & $1.32(0.84,2.09)$ & $1.12(0.69,1.82)$ \\
\hline age adjusted & 1.00 (reference) & $1.00(0.60,1.65)$ & $1.15(0.74,1.80)$ & $0.89(0.61,1.30)$ & $1.23(0.77,1.96)$ & $1.11(0.68,1.81)$ \\
\hline \multicolumn{7}{|l|}{ Waist to hip ratio $(\geq 0.85 v<0.85)$} \\
\hline cruac & 1.00 (reference) & 1.45( & 0.96( & $0.74(0$ & 1.20 & $2.17(1.18,3.98)$ \\
\hline age adjusted & 1.00 (reference) & $1.31(0.68,2.56)$ & $0.91(0.53,1.56)$ & $0.68(0.40,1.14)$ & $0.97(0.50,1.88)$ & $2.23(1.15,4.30)$ \\
\hline \multicolumn{7}{|c|}{ Age at first pregnancy ( $<20$ years $v \geqslant 20$ years) } \\
\hline crude & 1.00 (reference) & $1.31(0.61,2$ & $1.34(0$ & $1.07(0.56$ & 2.46 & $1.78(0.73,4.35)$ \\
\hline age adjusted & 1.00 (reference) & $1.29(0.60,2.80)$ & $1.32(0.48,3.61)$ & $1.07(0.56,2.04)$ & $2.42(1.15,5.09)$ & $1.75(0.71,4.28)$ \\
\hline \multicolumn{7}{|l|}{ Smoking (ever $v$ never) } \\
\hline $\begin{array}{l}\text { crude } \\
\text { age adjuste }\end{array}$ & $\begin{array}{l}1.00 \text { (reference) } \\
1.00 \text { (reference) }\end{array}$ & $0.86(0.54,1.37)$ & $1.21(0.77,1.91)$ & $1.09(0.74,1.60)$ & $0.69(0.42,1.15)$ & $1.31(0.79,2.17)$ \\
\hline \multicolumn{7}{|c|}{ Physical activity (<5730 kcal/kg/y $v \geqslant 5730 \mathrm{kcal} / \mathrm{kg} / \mathrm{y})$} \\
\hline crude & 1.00 (reference) & $0.87(0.48,1.59)$ & $0.67(0.39,1$ & $1.38(0.92,2.08)$ & $1.97(1.19,3.27)$ & $1.15(0.65,2.04)$ \\
\hline age adjusted & 1.00 (reference) & $0.73(0.39,1.35)$ & $0.56(0.32,0.98)$ & $1.36(0.86,2.16)$ & $1.74(0.91,3.30)$ & $1.12(0.60,2.09)$ \\
\hline \multicolumn{7}{|l|}{ Ever high blood pre } \\
\hline crude & 1.00 (reference) & $1.24(($ & 1.27( & $.75(\mathrm{C}$ & 1.44 & 1.16 \\
\hline age adjusted & 1.00 (reference) & $0.93(0.32,2.71)$ & $1.33(0.46,3.79)$ & $0.64(0.28,1.47)$ & $0.98(0.34,2.83)$ & $1.24(0.14,3.70)$ \\
\hline \multicolumn{7}{|c|}{ Total cholesterol ( $\geqslant 200 \mathrm{mg} / \mathrm{dl} v<200 \mathrm{mg} / \mathrm{dl}$ ) } \\
\hline crude & 1.00 (reference) & 0.8 & 0.86 & $1.20(0.77,1.87)$ & 1.4 & 9) \\
\hline $\begin{array}{l}\text { age adjusted } \\
\text { LDL cholesterol }(\geqslant 130 \mathrm{mg} / \mathrm{dl} v\end{array}$ & 1.00 (reference) & $0.70(0.35,1.43)$ & $0.81(0.42,1.60)$ & $1.16(0.72,1.88)$ & $1.20(0.65,2.20)$ & $1.03(0.56,1.91)$ \\
\hline \multicolumn{7}{|c|}{ LDL cholesterol ( $\geqslant 130 \mathrm{mg} / \mathrm{dl} v<130 \mathrm{mg} / \mathrm{dl}$ ) } \\
\hline $\begin{array}{l}\text { crude } \\
\text { age adjusted }\end{array}$ & $\begin{array}{l}1.00 \text { (reference) } \\
1.00 \text { (reference) }\end{array}$ & $\begin{array}{l}1.18(0.68,2.04) \\
1.03(0.57,1.86)\end{array}$ & $\begin{array}{l}0.98(0.57,1.68) \\
0.93(0.53,1.65)\end{array}$ & $\begin{array}{l}1.41(0.94,2.12) \\
1.38(0.89,2.15)\end{array}$ & $\begin{array}{l}1.43(0.84,2.42) \\
1.22(0.70,2.14)\end{array}$ & $\begin{array}{l}1.67(0.99,2.82) \\
1.64(0.93,2.91)\end{array}$ \\
\hline \multicolumn{7}{|c|}{ Post-load glucose ( $\geqslant 88 \mathrm{mg} / \mathrm{dl} v<88 \mathrm{mg} / \mathrm{dl}$ ) } \\
\hline & rence) & & & & 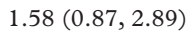 & 4) \\
\hline age adjusted & 1.00 (reference) & $0.63(0.34,1.17)$ & $0.48(0.28,0.84)$ & $0.96(0.62,1.50)$ & $1.37(0.72,2.63)$ & $0.88(0.49,1.58)$ \\
\hline
\end{tabular}

${ }^{\star}$ Odds ratios as estimated by generalised estimating equation (GEE) models using a logit link. $95 \%$ confidence intervals based on robust variance estimates. $\mathrm{NWC}=$ non-working class, WC=working class.
†Estimates for association of cross class categories with body mass index, age at first pregnancy, physical activity, and total cholesterol from models excluding NLF:WC

neutral household class measure and weakest for the individual level class measure, even after adjusting for age and marital/partner status (table 5). This pattern was apparent for: self rated health, height, BMI, age at first pregnancy, and glucose levels. For example, using the gender neutral household class measure, working class women were 1.9 times ( $95 \%$ confidence intervals $(\mathrm{CI})=1.1,3.4$ ) more likely than non-working class women to state their health was "fair or poor" (adjusted for age and marital status); the corresponding odds ratios for women categorised by the conventional household and individual class measures were, respectively, $1.5(95 \% \mathrm{CI}=0.9,2.5)$ and $1.3(95 \% \mathrm{CI}=0.8,2.2)$; analogous odds ratios for having a high post-load glucose level were, respectively, $1.7(1.1,2.6), 1.2(0.8,1.7)$, and $1.3(0.9,1.8)$. For two outcomes, waist to hip ratio and high blood pressure, effect estimates were comparably strongest for the gender neutral and conventional household class measures; for one outcome, being sedentary, effect estimates were equally greatest for the gender neutral household and individual class measures, while estimates of class effects were marginally greatest for the individual level class measure for only two related outcomes, total and LDL cholesterol. Only for one outcome, current smoking status, were no class effects observed, regardless of class measure used. Lastly, effect estimates generated with the combined household class measure, comparing women who were individually working class with no partners to women who were individually non-working class with non-working class partners, were similar to those yielded by the dichotomous gender neutral household class measure (comparing women in working class to non-working class households) (table 6). Their precision, however, was much less, because of smaller strata size.

Discussion

Our investigations of class inequalities in US women's health, considering a broad array of health outcomes, provide empirical evidence that it is feasible and desirable for public health studies to obtain data on the class position of soth women and their partners, if any. More, in nthroometric, metabolic, and self reported healt outcomes, class disparities were greatest when women were categorised by the gender neutra household class measure and smallest when using the individual level class measure. Finer stratification by the combined household class measure was less informative, as smaller cell size reduced precision of estimate effects.

OSSIBLE BIASES OR LIMITATIONS AFFECTING OUR STUDY

Before comparing our results to those of previous investigations or considering the broader mplication of our findings, it is useful to conider possible biases or limitations affecting in any epidemiological study, are problems affecting measurement of 
determinants and outcomes, and also those arising from choice and size of study population. Internal validity of our results, however, would not be affected by study cohort composition.

Firstly, our study was able to examine class inequalities in health using only the crude categories of "working class" and "non-working class". Given a relatively small sample size, we thus could not meaningfully compare health status among sub-groups of the "non-working class" (for example, business owners $v$ self employed $v$ professionals $v$ other supervisory employees) nor could we compare health status of skilled $v$ non-skilled workers. Our crude dichotomy, however, compares favourably with a related class dichotomy used in many studies demonstrating powerful socioeconomic inequalities in health: manual $v$ non-manual. divide, our approach does not blur class distincdivide, our approach does not blur class distinc-
tions among women, as working class women tions among women, as working class women
are more likely than men to be in non-manual are more likely than men to be in non-manual working class occupations (for example, secre-
taries, waitresses)..$^{10} 54$ Although a more refined taries, waitresses) ${ }^{2}{ }^{10}{ }^{54}$ Although a more refined have detected more nuanced socioeconomic gradients in health, our simple class typology none the less was capable of demonstrating that estimates of class inequalities in women's health vary depending upon individual and household class approach used to categorise women as "working class" and "non-working class".

Also limiting our ability to describe fully the range of socioeconomic disparities in the Women Twins cohort was a lack of other pertinent non-occupational socioeconomic data relevant for understanding women's health across the lifecourse, for example, childhood and adult data on income, wealth (including home or car ownership), and deprivation. ${ }^{16-28335556}$ Also lacking were data on gender disparities in access to household resources. ${ }^{15758}$ Moreover, while data on each respondent's educational level were obtained, comparable data on the partner's educational level were not collected. Thus, use of educational data would have been subject to the same methodological issues that were the focus of our class oriented investigation: whether individual or household level of education is the more salient measure to capture education related inequalities in health. To our knowledge, only one Finnish study ${ }^{35}$ has examined this question; whether results of this investigationwhich found equally strong effects for women's and spouse's education on Finnish women's mortality rates - are salient for US women is unknown. Selection of our class measures, however, was appropriate given our fundamental analytic question regarding appropriate occupation-based measures of class location for women, conceptualised in relation to production. We chose these theoretically grounded measures because social relations expressed through labour market position importantly determine (that is, are materially and logically prior to) possibilities for accumulation and consumption and may also be directly in the pathway to poor health (in relation to control over work and exposure to occupational hazards). ${ }^{11} 335960$ Further testing of the robustness of our findings could be accomplished by comparing gradients in health detected with analagous individual and household measures constructed using other social class schema (see, for example, socioeconomic measures employed in references ${ }^{1710185455}$ ).

Methods of collecting health data were unlikely to have biased detection of class nequalities in health in our cohort. Seven of our 11 health outcomes were based on results btained from physical examination or laboraintocols, with measurements conducted blind to th women's self reported socioeconomic position. Class related bias stemming from possible class differentials in recall for the four self reported health outcomes (general health status, age at first pregnancy, physical activity, and current moking status) was likely to have been conomic position at the end of the self adminstered questionnaire, after health related queries. Analysing data on diverse health outcomes obtained by different methods, moreover, permitted examining patterns of class gradients across a broad spectrum, thereby reducing the ackelihood that results reflected only select associations between class location and particular health outcomes.

Ability of our study to detect class inequalities in health, however, as well as generalisability of our results, may have been diminished by selection bias. This is because our sample consisted of members of a health maintenance organisation who chose to enroll in the Kaiser Permanente Women Twins Study. All respondents thus had access to health care and, because they agreed to be in the study, may have been prone to exhibit health conscious behaviour. In fact, women in our cohort had better health than women in the general US population for at least several health indicators. For example, among women 45 to 64 years old, " the per cent reporting "fair" or "poor" health status in the US in 1990 was 16 per cent (page $181)^{61}$ versus 8.4 per cent in our cohort. Similarly, 35 per cent of US white women 20 to 47 years old in 1990 were categorised as obese $\left(\mathrm{BMI}>27.3 \mathrm{~kg} / \mathrm{m}^{2}\right)$ (page 192), ${ }^{61}$ compared with 27 per cent in the Women's Twins cohort. Cigarette smoking was also less pr and 22 per cent, respectively, of US women 35-44, 45-64 and 65 years and older were current cigarette smokers in 1990 (page 182), ${ }^{61}$ corresponding proportions among the twins were 18,17 , and 9 per cent. Lastly, both high blood pressure and high cholesterol were about two times more prevalent in the genera (ins pproximately 20 per cent of US wome to 74 years old (in 1988-1994) (page 190-2), ${ }^{61}$ versus under 10 per cent of the twins. Thus, class inequalities in health detected in our study may be truncated compared with what might have been observed in a more representative sample of US women. Other factors 
originally residing in the San Francisco Bay Area, and an inability to distinguish between heterosexual and lesbian households. Conceivably, discrimination, differential labour force participation rates, and alternative household structures could modify effects observed using the individual and household class measures used in this study ${ }^{12762}$; ascertaining whether such effect modification exists will require additional empirical investigation because no studies, to our knowledge, have explored this topic. Similarly, relatively small sample size precluded us from conducting meaninful analyses of whether observed patterns of class effects differed by age, comparing women of employment age to women at retirement age.

COMPARISON TO OTHER STUDIES: WOMEN, CLASS, AND INEQUALITIES IN HEALTH

Lending confidence to interpretation of our study results are findings of prior investigations on class and women, including class inequalities in health. Taken together, they bolster the view that: (a) information about women's class location will be maximised by taking into account the class position of the woman and her partner or other head of household, if present, (b) both individual and household class are pertinent measures of class position, depending on the outcome being studied, and (c) it is protective to live in a household in which at least one adult is "non-working class", whether or not this adult is the respondent.

Firstly, with regard to class distribution of women, a set of coordinated studies conducted in the early 1980s included data on class position, as determined by Wright's typology, of wives and husbands from four different countries: the United States (550 couples), Sweden (672 couples), Norway (960 couples), and Canada (690 couples) (page 223-36). ${ }^{11}$ Within the United States, 57 per cent of women in these dual earner households were individually classified as working class, while lower proportions would have been classified as working class using a conventional household approach (44 per cent) and a gender neutral household approach (30 per cent) (page 226). ${ }^{11}$ Moreover, although the majority of dual earner families in all four countries were class concordant, 30 to 35 per cent husbands and wives occupied "clearly different class locations", of whom half had one spouse in the working class while the other was either "an employer, an expert manager, a manager or a professional" (page 227). ${ }^{1}$ A subsequent smaller US study of 101 women (51 black, 50 white) residing in Alameda County, CA in 1987 that used a modified version of Wright's class typology likewise reported the proportion of women categorised as working class equalled 56 per cent for the individual class measure, compared with 43 per cent using a gender neutral household class measure; in this sample, only 4 per cent of women were not in the paid workforce. ${ }^{36}$ Similar shifts in proportions of women categorised as working class, using the individual and different household class measures, are evident cases, a more rigorous definition of "working class" household was provided by the gender neutral as compared with conventional household class measure, as the former includes only ouscholds in which at least one adult is "working class" and all others are either "working class" or "not in the paid labour force".

Secondly, with regard to class inequalities in women's health, only three epidemiologica studies comparing occupation-based individual and household class measures have included outcomes equivalent to those examined in our investigation 313336 None, however, ince in our investigation 35 None, however, presented data in a form that could be compared directly to our results. The small US study of 51 black and 50 white women, for example, included age at first pregnancy among its reproductive health outcomes, but models examining effects associated with individual and gender neutral household class also vicluded data on race/ethnicity, age, and dhree r household class, were associated with age at first pregnancy. The two relevant British studies, in turn, examined self reported health status among married women. ${ }^{313}$ One, based on women age 65 and older included in the 1985 class gradients for reporting less than good health (adjusting for age) for both the individual and conventional household class measures. ${ }^{31}$ By contrast, the study based on women 20 to 59 years old included in the 1991-1922 British General Household survey, reported that in models adjusting for the womn's age and educational level, partner's class was more strongly and consistently associate with having "less than good" health than the women's individual class. ${ }^{3}$

The handful of other relevant epidemiological studies, conducted among women in the UK, focused on mortality ${ }^{9364}$ and also self reported longstanding limiting illness. ${ }^{29-31}$ These investigations likewise reported stronger ssociations between married women's health and their husbands' class versus their own class. A recent study of women's mortality in Finland, however, reported comparable clas effects for women whether categorised by their own or their spouse's occupation. ${ }^{35}$ Given greater labour force participation by women in ave been interpreted as suggesting that as women's labour force participation increases, so too may the significance of women's individual class as a predictor of women's health status ${ }^{33} 3566$; whether wives' individual class is increasingly predictive of husband's health status remains to be explored.

ECOMMENDATIONS FOR MEASURING CLASS TEOUALTIES IN WOMEN'S HEALTH

In conclusion, we suggest that epidemiological studies concerning class inequalities in women's health should use the gender neutra household class measure or, if sample size is sufficiently large, the combined household class measure. Reliance upon solely individual evel class data is likely to result in underestimation of class inequalities in women's health 
and also lead to the erroneous conclusion that class position is less strongly associated with women's as compared with men's health.

In addition to these methodological points, our results underscore the continued relevance of theoretically grounded occupationbased class measures for public health research, as such measures not only discriminate between classes of people with better and worse health but also usefully illuminate social relations that underlie class structure and drive class inequalities in health. ${ }^{1159606768}$ Ironically, to the extent that women's rising labour force participation reflects a growing necessity for families to have two (or more) wage earners to be financially secure, ${ }^{10}$ quantifying class inequalities in women's health may increasingly require measuring not only women's individual class but also their household class and socioeconomic conditions in their neighbourhoods and broader polity. Doing so will probably improve description Doing so will probably improve description
and analysis of social inequalities in health, thereby expanding data to inform action and policies to reduce social inequalities in health.

The authors thanks Eugenie Coakley and Bix Swain for their Twins Study data files from the Division of Research to the Harvard School of Public Health and Akiko Okamoto for her

Funding:this investigation was supported by FIRST investigator Lung and Blood Institute.
Conflicts of interest: none.

1 Krieger N, Williams DR, Moss NE. Measuring social class

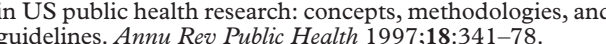
2 Haug MR. Social class measurement and women's occupa-
tional roles. Social Forces 1973;52:87-99.
3 Acker JR. Women and social stratification. Am f Sociol 1973,78:2-48. Britten N, Heath A. Women, men, and social class. In: Gar-
marnikow E, Morgan DH, Purvis J, et al, eds. Gender, class, marnikow E, Morgan DHJ, Purvis J, et al, eds. Gender, class, 5 Goldthorpe JH. Women and class analysis: in defense of the 6 Stanworth M. Women and class analysis: a reply to John Goldthorpe. Sociology 1984;18:159-70.

7 Dale A, Gilbert GN, Arber S. Integrating women into class Macfarlane A. Official statistics and women's health and ness. In: Roberts $\mathrm{H}$, ed. Women's health counts. Londo-

9 Mouser KA, Pugh HS, Goldblatt PO. Inequalities in women's health: looking at mortality differentials using an alternative approach. BMF 1988;296:1221-4.
Sorensen A. Women, family and class. Annu Rev Sociol 1994;20:27-47.
Sorensemily and class. Annu Reo Socol 11 Wright EO. Class

Cambridge: Cambridge University Press, 1997. Maclntyre S, Hunt K. Socio-economic position, gender,
and health: how do they interact? 7 Health Psychology 1997 ; 2:315-34.

13 US Bureau of the Census. Statistical Abstract of the United States: 1997. 117th ed. Washington, DC: US Departmen 4 Franck I, Browstone E. The women's desk reference. New York: Viking, 1993.
15 Koditschek T. The gendering of the British working class.
Gender and History 1997:9:333-63. 16 Rowbotham S. A century of women: the history of women in
Britain and the United States. New York: Viking, 1997 .
17 Charles N. Women and class-a problematic relationship? Charles N. Women and class - a problematic relationship? Marshall G, Roberts S, Burgoyne C, et al. Class, gender, and the assymetry hypothesis. European Sociological Review
1995;:11-15. 1995;1:11-15.
Abbott P. Sapsford R. Women and social class. London: 19 Abbott P, Sapsford R. Women and social class. London: 20 Baxter I. Gender and class analysis: the position of women
in the class structure. Australia New Zealand fournal of Soci21 Zipp JF, Plutzer E. Wives and husbands: social class, gender, and class identification in the United States. Sociology
22 Hout $M$. The association between husbands' and wives' occupations
$397-409$ identity in the United States, Sweden, Norway an

4 Heath A, Britten N. Women's jobs do make a difference: a 5 Davey Smith G, Hart C, Hole D, et al. Education and occupational social class: which is the more important indicato 153-60.

class in epidemiology, Epidemiol Rev 1988;10:87-121.
chial dilliams DR, Collins C. US socioeconomic and racial Sociol 1995;21:349-86.

28 Marmot M, Bobak M, Smith GD. Explanations for socia inequalities in health. In: Amick B III, Levine S, Tarlov AR, Press, 1995:172-210. 29 Arber S. Social class, non-employment, and chronic illness:

Arber S. Gender and class inequalities in health: under-
standing the differentials. In: Fox J, ed. Health inequalities European countries. Aldershot, UK: Gower, 1989:250-79. Arber S, Ginn J. Gender and inequalities in health in late life. Soc Scl Med 1993;36:33-46. Arber S. Class, paid employment and family roles: making
sense of structural disadvantage, gender, and health status.
Soc Sci Med 1991;32:425-36.

33 Arber S. Comparing inequalities in women's and men's
health: Britain in the 1990s. Soc Sci Med 1997;44:773-87. Chandola T. Social inequality in coronary heart disease: a
comparison of occupational classifications. Soc Sci Med

Socioeconomic mortality differentials in men and women according to own and spouse's character-
istics in Finland. Sociology of Illness and Health 1995;17: $353-75$

Krieger N. Women and social class: a methodological study comparing individual, household, and census measures as
predictors of black/white differences in reproductive history. I Epidemiol Community Health 1991;45:35-42. women the Norwegian experience. Sociology of Illness and Health 1991;13:492-50

38 Selby JV, Newman B, King MC, et al. Environmental and women: a matched co-twin analysis. Am $\mathcal{f}$ Epidemiol 1987; 125:979-88.

39 Krieger N, Okamoto A, Selby JV. Adult female twins' recal validation study for public health research. Am $¥$ Epidemi 1998; 147:704-8. Friedman GD, Lewis AL. The Kaiser Permanente twin reg-
istry. In: Nance WE, ed. Twin research: part B, biology and epidemiology. New York: Alan R Liss, 1978:173-7. 1 Wright EO, Costello C, Hacken D, et al. The American cla

structure. Am Sociol Rev 1982;47:709-26. health chartbook. Health, United States, 1998. Hyattsville, Dreve National Center for Health Statistics, 1998 . supplement. Series DS No. 15. London: The Stationary

fat intake and insulin concentrations in healthy women twins. Diabetes Care 1993;16:1459-69.

Daskell WL, et al. Comparison of two methods of assessing physical activity in the Coronary Artery Risk Development in Young Ad
Study. Am I Epidemiol 1991; 133:1232-45.

Maver EJ, Newman B, Austin MA, et al. Genetic and environmental influences on insulin levels and the insulin Epidemiol 1996;143:323-32. Eorld Health Organization. Physical status: the use and
interpretation of anthropometry. Geneva: World Health 8 SAS Institute, Inc. SAS for UNIX Release 6.12.Cary NC, 1996.

data with, Feinleib M, Hubert H. Regression analysis of data with correlated errors: an exa
study. $\mathcal{F}$ Chron Dis $1985 ; 38: 165-70$.

50 Liang KY, Zeger SL. Longitudinal data analysis using gen-

1 Zeger SL, Liang KY. Longitudinal data analysis for discrete and continuous outcomes. Biometrics 1986;42:121-30. Carey VJ. Implementation of gee() for S-PLUS, version
4.13 (Jan 26, 1998). Boston, MA: Harvard University. Available from Statlib at http://lib.stat.cmu.edu/S/ 3 Townsend P, Davidson N, Whitehead M. Inequalities in
health: The Black Report and the health divide. London: PenEvans G. Putting assessment of the cross-sex validity of the Goldthorpe clas

schema. Sociology 1996;30:209-34.
Graham H, Blackburn C. The socio-economic patterning of health and smoking behaviour among mothers with young
children on income support. Sociology of Illness and Health
$1998 ; 20: 215-40$. 
56 Kuh $\mathrm{D}$, Ben-Shlomo Y, eds. A lifecourse approach to chronic
disease epidemiology: tracing the origins of ill-health from early 57 Doyal L. What makes women sick? Gender and the political economy of health. New Brunswick, NJ: Rutgers University
Press, 1995 Thomas D. Like father, like son; like mother, like daughter parental resources and child height. Fournal of Human Resources 1994;29:950-88.
Wright EO. The class analysis of poverty. Int $\mathcal{F}$ Health ServWres 1995;25:81-100.
inalys of poverty. In 60 Navarro V. Crisis, health, and medicine: a social critique. New
York: Tavistock, 1986 .
61 National Center for Health Statistics. Health, United States

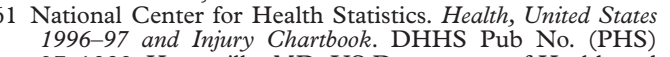
97-1232. Hyattsville, MD: US Department of Health an Human Services, 1997.
Krieger N. Discrimination and health. In: Berkman L,
Kawachi I, eds. Social epidemiology. Oxford: Oxford UniverKawachi I, eds. Soci
sity Press (in press).
63 Pugh H, Moser K. Measuring women's mortality differences. In: Roberts $\mathrm{H}$, ed. Women's health counts. London:
Routledge, 1990:93-112. 64 Moser K, Pugh H, Goldblatt P. Mortality and the social classification of women. In: Goldblatt $\mathrm{P}$, ed. Longitudinal
study: mortality and social organization. London: HMSO, 1990: 145-62. 6 Arber S. Revealing women's health: re-analysing the
General Household Survey. In: Roberts H, ed. Women's General Household Survey. In: Roberts $\mathrm{H}$,
health counts. London: Routledge, 1990:63-92 health counts. London: Routledge, 1990:63-92
6 Arber S, Lahelma E Enequalities in women's and men's ill-
health: Britain and Finland compared. Soc Sci Med 67 Krieger N, Fee E. Social class: the missing link in US health 67 Krieger N, Fee E. Social class: the mitas
data. Int $\mathcal{F}$ Health Serv 1994;24:25-44.

68 Krieger N, Fee E. Measuring social inequalities in health in
the United States: an historical review, 1900-1950. Int $\mathcal{F}$ Health Serv 1996;26:391-41

Amott T. Caught in the crisis: women and the US economy
today. New York: Monthly Review Press, 1993 .

Appendix 1 Elaborated class typology developed by Erik Olin Wright (pages 20-25) ${ }^{11}$

\begin{tabular}{|c|c|c|c|c|c|}
\hline \multirow{2}{*}{$\begin{array}{c}\text { Number of } \\
\text { employees } \\
\text { Many }\end{array}$} & \multirow{6}{*}{$\begin{array}{l}\text { Owner } \\
\text { (1) } \\
\text { Capitalist } \\
\text { (2) } \\
\text { Small employer } \\
\text { (3) } \\
\text { Petty bourgeoisie }\end{array}$} & \multicolumn{3}{|c|}{ Relation to means of production } & \multirow[b]{2}{*}{ a to authority } \\
\hline & & & Employees & & \\
\hline & & Expert manager & Skilled managers & Nonskilled managers & Managers \\
\hline Few & & (7) & & (9) & \\
\hline \multirow{3}{*}{ None } & & $\begin{array}{l}\text { Expert supervisor } \\
\text { (10) }\end{array}$ & $\begin{array}{l}\text { Shilled supervisors } \\
\text { (11) }\end{array}$ & $\begin{array}{l}\text { Nonskilled supervisors } \\
\text { (12) }\end{array}$ & Supervisors \\
\hline & & Exper & Skilled workers & Nonskilled workers & Non-management \\
\hline & & $\begin{array}{l}\text { Expert } \\
\text { Relation to scarc }\end{array}$ & $\begin{array}{l}\text { Skilled } \\
\text { skills }\end{array}$ & Nonskilled & \\
\hline
\end{tabular}

The 12 locations in Wright's typology reflect social relations among people expressing: (a) relation to means of production (owne $v$ employee), (b) relation to authority (managers who are involved in organisational decision making; supervisors who have powe experts $v$ non-experts); also relevant is number of employee ${ }^{11}$ In Wright's typology cells 1 and 2 accordingly represent capitalist locations, cell 3 is the location of the petty bourgeoisie (self employed), cells 4 through 10 occupy what Wright terms "contradictory class locations" as "... they can be considered simultaneously in the capitalist class and in the working class: they are like capitalis in that they dominate workers; they are like workers in that they are controlled by capitalists and exploited within production")," while cells 11 and 12 are working class locations. 